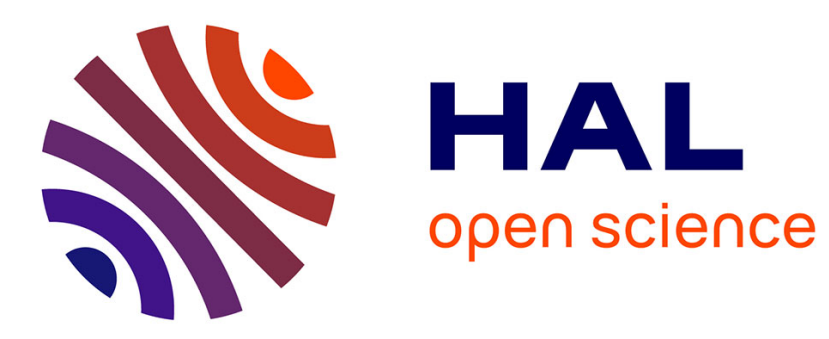

\title{
Suivi médical de salariés exposés au beryllium
}

G. Thin, Mireille Matrat, J. C. Duché, Florence Lauzier, Michel Goudal, R. Quang, Catherine Hubert, Jean-Pierre L'Huillier, Jérôme Barré, Jean-Claude Pairon, et al.

\section{- To cite this version:}

G. Thin, Mireille Matrat, J. C. Duché, Florence Lauzier, Michel Goudal, et al.. Suivi médical de salariés exposés au beryllium. Archives des Maladies Professionnelles et de L'Environnement, 2009, 70 (4), pp.395-404. 10.1016/j.admp.2008.11.001 . inserm-00428562

\section{HAL Id: inserm-00428562 https://www.hal.inserm.fr/inserm-00428562}

Submitted on 29 Oct 2009

HAL is a multi-disciplinary open access archive for the deposit and dissemination of scientific research documents, whether they are published or not. The documents may come from teaching and research institutions in France or abroad, or from public or private research centers.
L'archive ouverte pluridisciplinaire HAL, est destinée au dépôt et à la diffusion de documents scientifiques de niveau recherche, publiés ou non, émanant des établissements d'enseignement et de recherche français ou étrangers, des laboratoires publics ou privés. 


\section{SUIVI MEDICAL DE SALARIES EXPOSES AU BERYLLIUM :}

Medical follow-up of beryllium - exposed workers :

\section{G Thin ${ }^{1}$, M Matrat ${ }^{1,2}$, JC Duché ${ }^{3}$, F Lauzier $^{4}$, M Goudal $^{4}$, R Quang $^{5}$, C Hubert ${ }^{1}$, JP L'Huillier ${ }^{1}$, J Barré ${ }^{3}$, JC Pairon ${ }^{1,2}$ C de Clavière ${ }^{1}$}

1 CHI Créteil, unité de pathologie professionnelle, Service de pneumologie et de pathologie professionnelle 94000 CRETEIL, 2 INSERM U 955, 94000 CRETEIL, 3 Unité fonctionnelle de pharmacologie-toxicologie CHI Créteil 94000 CRETEIL, 4 CRAMIF Service HIPP Place de l'Argonne 75019 PARIS, 5 ACIST, 94550 Chevilly Larue.

Auteur correspondant : e-mail cdeclaviere@free.fr

\section{Summary :}

Purpose of the study: determination of a systematised procedure for medical follow-up of beryllium-exposed workers.

Method: a medical follow-up of workers from a factory machining beryllium (Be) either plain or as an alloy started in 2001. Be Lymphocyte Proliferation Tests (LPT) were performed for screening Be sensitisation and were calculated again according to 1142-2001 speciation of the American Department Of Energy. A working group included occupational physicians, specialists in occupational prevention and biologists and established based on a review of medical literature an algorithm for medical folllow-up of workers. This algorithm determines how to handle medically such workers and is mostly based upon Be LPT results.

Results: 14 workers were followed for 6 years at the most. Abnormal (positive or border-line) tests were noticed in 4 of them. These tests became negative within 3 to 18 months in 3 of them, in parallel with Be working decrease and with strengthening of preventive measures. Neither respiratory, nor radiological, nor functional abnormal symptom occurred in the $4^{\text {th }}$ worker that could evoke an out coming chronic berylliosis. 
Discussion and conclusion: This shows how necessary it is to standardise Be LPT method and results and how important it is to establish guidelines for a long-term follow-up of workers who may be exposed to Be and to strengthen preventive measures. Key words: beryllium, sensitisation, occupational exposure, prevention, Lymphocyte Proliferation Test

\section{RESUME}

Objectif : Détermination d’une démarche systématisée pour le suivi médical de salariés exposés au béryllium.

Méthode: Un suivi médical de salariés d'une entreprise du secteur de l'usinage du béryllium (Be) pur ou en alliage a été initié en 2001. Des tests sanguins de prolifération lymphocytaire au béryllium (TPL Be) utilisés dans le dépistage d'une sensibilisation au Be, avec recalcul selon la spécification 1142-2001 du département de l'énergie américain ont été réalisés. Un groupe de travail incluant médecins du travail, préventeurs et biologistes, a établi un algorithme décisionnel de suivi médical de ces salariés, à partir d’une revue de la littérature. L’algorithme déterminant la prise en charge à instaurer repose principalement sur les résultats du TPL Be.

Résultats : 14 salariés ont été suivis pendant au plus 6 ans. Le TPL Be a été trouvé anormal chez 4 d'entre eux (tests positifs ou « limites ») puis s’est négativé pour 3, en l'espace de 3 à 18 mois, parallèlement à une diminution de l'activité Be et à un renforcement des mesures de prévention. Aucune symptomatologie respiratoire clinique, radiologique ou fonctionnelle laissant suspecter une évolution vers une bérylliose chronique n’est apparue pour le quatrième.

Discussion et conclusion : Outre la nécessité de standardiser la technique du TPL Be et l’expression de ses résultats, il apparaît important d'établir des recommandations de 
surveillance médicale pour le suivi à long terme des salariés susceptibles d’être exposés au Be et de renforcer les mesures de prévention.

Mots clés : béryllium, sensibilisation, exposition professionnelle, prévention, Test de Prolifération Lymphocytaire

\section{Introduction}

Le béryllium (Be) est un métal léger, dur, résistant et bon conducteur d'électricité et de chaleur. Il est utilisé soit en poudre (oxyde de Be) soit sous forme de métal pur ou dans des alliages, essentiellement dans l'industrie nucléaire, aéronautique ou aérospatiale, dans l'industrie d'appareillage électrique et électronique, dans la construction mécanique, dans l'industrie des instruments de précision (horlogère par exemple), dans le raffinage des métaux précieux et dans la fabrication de matériaux réfractaires, de verreries d'art ou de prothèses dentaires (1). L'exposition au Be concerne 9400 à 14400 salariés en France dans les secteurs de la mécanique, des fonderies de métaux précieux et à la fabrication de moules dans le secteur de la plasturgie (2). Les métiers exposant le plus à l'inhalation de Be sont ceux comportant l'usinage du Be ou de ses alliages (1).

Une revue de la littérature en langue française a été réalisée en 2005 sur la bérylliose chronique (3). La pénétration du Be se fait essentiellement par voie respiratoire en milieu professionnel ; par voie cutanée, elle est limitée en peau saine mais favorisée en cas de lésions (4). Le poumon est l'organe cible du Be inhalé. Du fait d’un mécanisme immunologique, il se produit une sensibilisation au Be qui peut conduire, en fonction de déterminants incomplètement élucidés, à une bérylliose chronique. Il s'agit d'une granulomatose systémique à prédominance pulmonaire. Sa symptomatologie clinique et radiologique est proche de celle de la sarcoïdose médiastino-pulmonaire mais son 
pronostic est plus sévère. Le lavage broncho-alvéolaire met en évidence une alvéolite lymphocytaire avec prédominance des lymphocytes T4. La bérylliose chronique peut évoluer vers une insuffisance respiratoire chronique (5). La mise en évidence, dans des études épidémiologiques, d’une augmentation du taux de mortalité par cancer bronchopulmonaire, après exposition à de fortes concentrations de Be, est à l'origine de la classification du Be en produit cancérogène par l’union européenne (catégorie 2, R49) et par le CIRC (groupe 1) (6).

Il existe peu de données sur le suivi longitudinal des salariés exposés au Be. La question se pose de la surveillance médicale à proposer en cas d'exposition professionnelle au Be. En 2001, nous avons initié un suivi médical de salariés d’une entreprise d'usinage de pièces métalliques, exposés au Be. Cette démarche a impliqué un groupe de travail pluridisciplinaire. Nous présentons ici les résultats de ce suivi.

\section{Matériel et méthodes}

L’entreprise concernée travaille dans le secteur de l'aéronautique et emploie environ 60 salariés. Quatorze d'entre eux usinent des pièces en métal ou alliage, dans un ou plusieurs ateliers. Les pièces sont à base de Be pur ou en alliage avec de l'aluminium (30\%). Les activités qui exposent au Be comportent l'usinage (tournage et fraisage) effectué dans un atelier spécifique, et, dans une moindre mesure, l'électroérosion, l'assemblage au cours duquel les pièces peuvent être brasées, le traitement de surface ou l'attaque chimique des pièces, le contrôle qualité des pièces et enfin l'analyse des prélèvements atmosphériques ou surfaciques.

Parmi les salariés exposés au Be, il y a 7 fraiseurs, 2 ajusteurs, 3 techniciens, un chef de fabrication /contrôleur et un tourneur. L’activité Be a pu occuper ces salariés au moins 2 
à 4 semaines par an et au plus 5 mois par an.

Les conditions de travail sont globalement satisfaisantes. Un effort particulier est réalisé afin de mettre en place des mesures de prévention collective et individuelle adaptées.

Ainsi, tournage et fraisage de pièces comportant du Be sont exécutés sur des machines à commande numérique, complètement capotées. Le travail est réalisé « à l’humide », avec des huiles de coupe pour l'alliage, et le plus souvent à sec pour le Be pur, dans une machine étanche avec captage à la source et mise en dépression. En fin d'usinage, chaque pièce doit être décontaminée par trempage dans l'alcool avant réalisation d'un frottis de contrôle systématique.

La ventilation de l'atelier assure un renouvellement de l'atmosphère des locaux. Une aspiration est maintenue en permanence, pour éviter la remise en suspension de particules de Be fixées sur le système de ventilation.

Chaque poste de travail est équipé d'une buse d'aspiration orientable. L'ensemble des filtres est remplacé systématiquement tous les ans et la quantité de poudre de Be présente est alors vérifiée. Les filtres sont aussi changés en cas de colmatage ponctuel détecté par le dispositif de surveillance. Cette opération est réalisée par un opérateur de l'atelier d'usinage du Be, muni alors d'un équipement de protection individuelle spécial et formé à cette tâche. Le réseau des gaines d'aspiration est inspecté annuellement et décontaminé si nécessaire. Les déchets générés par l’activité Be sont stockés dans des fûts scellés avant transport puis destruction par une société spécialisée. En fin de journée de travail, un nettoyage de l'atelier est effectué et son efficacité est contrôlée par un frottis de surface. Il est renouvelé en cas de résultat anormal. Le sol des ateliers, vestiaire et machinerie est nettoyé deux fois par semaine, avec des aspirateurs réservés à cette opération et équipés d’une filtration adéquate. 
Sur le plan des mesures de prévention individuelle, les salariés portent des combinaisons fermées au col et aux poignets, des gants en coton initialement puis remplacé par du nitrile et des surchaussures, jetés après utilisation. Des protections respiratoires « filtrantes », de type P3 sont à leur disposition pour l'usinage du Be et en cas d'incident ponctuel dans le fonctionnement du système de ventilation - aspiration. Des masques isolants sont disponibles pour des opérations spécifiques à haut risque de contamination (changement de filtres, nettoyage des gaines, etc...). Les salariés de l'atelier usinage se lavent les mains en sortant de l'atelier et prennent une douche en fin de période de travail. Les visiteurs et les personnels qui ne travaillent pas dans l'atelier y entrent par l’intermédiaire d'un sas, toujours revêtus de survêtements jetables.

Le niveau d'exposition au Be est objectivé par des prélèvements atmosphériques d'ambiance et surfaciques, réalisés sur filtres d'acétate de cellulose, quotidiennement dans l'atelier d'usinage et de façon hebdomadaire dans d'autres ateliers. Par ailleurs, des prélèvements atmosphériques (d'ambiance ou individuels) sur 3 heures ont été faits ponctuellement par un laboratoire extérieur. La VME française pour le Be est $2 \mu \mathrm{g} / \mathrm{m}^{3}$. Il n’y a pas de valeur limite réglementaire française pour les mesures surfaciques de Be. L'entreprise considère que $3 \mu \mathrm{g} / \mathrm{dm}^{2}$ est la valeur surfacique limite à ne pas dépasser, au delà de laquelle une décontamination se justifie. Ce chiffre est retenu comme valeur justifiant une décontamination par le département de l'énergie des Etats-Unis, alors que le « Cardiff Atomic Weapons Establishment » du Royaume-Uni retient 1 $\mu \mathrm{g} / \mathrm{dm}^{2}$ (7). Les résultats des prélèvements atmosphériques d'ambiance, comparés à la VME française, montrent que dans l'atelier d'usinage du Be, ils sont inférieurs ou égaux à $1 / 20^{\text {ème }}$ de la VME, qu'il s'agisse de ceux faits par l'entreprise $(n=24)$ ou ponctuellement par un laboratoire extérieur $(n=3)$ et dont nous avons eu connaissance. 
En ce qui concerne les prélèvements atmosphériques individuels réalisés par le laboratoire extérieur, 2 sont inférieurs au dixième de la VME et 1 dépasse la VME (1,5 VME).

Les résultats des prélèvements surfaciques réalisés par l'entreprise disponibles pour 5 jours ( $n=60$ ) sont compris entre 0,09 et $15,5 \mu \mathrm{g} / \mathrm{dm}^{2}$, la médiane de chaque jour est inférieure à $3 \mu \mathrm{g} / \mathrm{dm}^{2}$. Les médianes sont inférieures à la valeur surfacique adoptée par l’entreprise et à la valeur de référence américaine.

Les salariés exposés au Be au sein de l'entreprise ont été adressés à un centre de consultation de pathologie professionnelle. Au cours de ces consultations des tests de prolifération lymphocytaire en présence de Be (TPL Be) ont été prescrits. En effet, le TPL Be constitue un marqueur d'effet biologique du Be. La prolifération lymphocytaire en présence de Be est évaluée par mesure de l'incorporation de thymidine tritiée (en coups par minute) qui est comparée à celle observée en présence de témoins négatifs (lymphocytes sans Be) et en présence de témoins positifs, mitogènes phytohémagglutinine (PHA) et pokewed mitogen (PWD) ou anatoxine tétanique qui mettent en évidence la bonne réactivité des cellules. Elle est exprimée par un index de stimulation calculé à partir du rapport entre le nombre de coups par minute obtenus en présence de Be et le nombre de coups par minute sans Be. La prolifération a été mesurée jusqu'en septembre 2006 à deux temps d'incubation (3 et 6 jours), et en présence, à chaque temps, de 4 concentrations de sel de Be (2, 20, 200 et $2000 \mu \mathrm{g} / \mathrm{ml}$ ). A chaque temps étaient réalisées plusieurs mesures de témoins négatifs et positifs (PHA, PWD). A l'époque, le test était alors considéré comme positif en présence d'au moins 2 index de stimulation supérieurs au seuil de positivité du test fixé à 2,5. En présence d’un seul index supérieur au seuil, on parlait de résultat « limite ». 
Depuis septembre 2006, selon la spécification 1142-2001 US du département de l'énergie américain de 2001, la prolifération lymphocytaire est mesurée à deux temps d'incubation (3 et 6 jours), et en présence, à chaque temps, de 3 concentrations de sel de Be (1, 10 et $100 \mu \mathrm{M})$ avec toujours, en parallèle, la réalisation de plusieurs mesures témoins (incluant en plus l'anatoxine tétanique) (8). Les résultats sont issus de calculs logarithmiques à partir des médianes des résultats de prolifération des lymphocytes en présence de Be comparées aux témoins et aboutissant à des index. Le test est considéré comme « positif » si au moins 2 index statistiques (IS) sur les 6 obtenus (3 à J3 et 3 à J6) sont supérieurs à 2,53 et si l'index biologique (IB) qui compare les IS statistiques à ceux obtenus dans une population de sujets normaux non exposés au Be est supérieur à 3,1. Le résultat du TPL Be est considéré comme «limite » si 2 IS au moins sont supérieurs à 2,53 mais que l'IB est inférieur à 3,1 ou si l'IB est supérieur à 3,1 et qu'il n’y a pas au moins 2 IS supérieurs à 2,53.

Si un résultat est « limite », un nouveau prélèvement est effectué 3 à 6 mois plus tard. Les résultats des tests effectués avant septembre 2006 ont été recalculés selon la nouvelle modalité et présentés selon les deux modes de calcul dans les résultats (tableau 1).

En 2005, un groupe de travail est constitué pour déterminer la stratégie de surveillance médicale à proposer. Ce groupe comporte le médecin du travail de l'entreprise, des médecins de la consultation de pathologie professionnelle, des biologistes qui réalisent les TPL et des membres du service de prévention des risques professionnels de la CRAMIF. Une revue de la littérature est faite en prenant en compte les références des bases de données médicales disponibles au $1^{\mathrm{er}}$ juillet 2005, et les présentations de la « conférence internationale de la recherche sur le béryllium » qui s’est tenue en mars 
2005 à Montréal (1).

Il s’avère qu'il existe très peu d'études longitudinales de suivi à long terme de sujets exposés au Be. Les données de la littérature sont essentiellement issues soit de cas isolés de sujets sensibilisés ou atteints de bérylliose chronique soit d’études transversales menées sur un site industriel précis (surtout chez des céramistes, dans l'industrie nucléaire ou dans des usines d'alliages au Be), mais sans suivi longitudinal. Les examens réalisés pour ces suivis sont présentés dans le tableau 2.

Des «recommandations pour l'évaluation d'un patient à risque de bérylliose chronique » ont été proposées par le groupe du National Jewish Medical and Research Center » (NJMRC) et un algorithme décisionnel a été élaboré par les Comités de maladies professionnelles pulmonaires du Québec à titre expérimental pour les travailleurs exposés au Be (9).

- Pour le NJMRC, l'arbre décisionnel débute lors de l’obtention de 2 TPL Be sanguins (analysés dans 1 ou 2 laboratoires). S’ils sont positifs, ils sont complétés par une évaluation clinique, une fibroscopie bronchique, un lavage bronchoalvéolaire, un TPL Be sur le lavage broncho-alvéolaire et des biopsies pulmonaires transbronchiques. Si les 2 TPL Be sanguins sont normaux, des anomalies cliniques, radiographiques ou portant sur la fonction respiratoire sont recherchées. En cas d'anomalie de ce bilan médical complémentaire, une fibroscopie bronchique, un lavage broncho-alvéolaire avec TPL Be et biopsies pulmonaires transbronchiques sont effectués. Si un seul TPL Be sanguin est positif, il est contrôlé. Le moment précis et la fréquence des examens paracliniques ne sont pas spécifiés.

- L’algorithme du Québec se fonde sur la réalisation d’un TPL Be sanguin adressé à 2 laboratoires. Si les résultats sont normaux, le TPL Be est répété tous les 2 à 5 ans. Si les 
résultats sont anormaux, une évaluation clinique, une radiographie pulmonaire, une tomodensitométrie thoracique et des explorations fonctionnelles respiratoires (EFR) sont réalisées. En cas d’anomalie, ils sont complétés par une fibroscopie bronchique avec lavage broncho-alvéolaire, TPL Be sur ce lavage broncho-alvéolaire et biopsies. Parallèlement au bilan, il existe des préconisations vis-à-vis de l'exposition au Be pour les sujets sensibilisés ou atteints de bérylliose chronique, allant de moins de $0,1 \mu \mathrm{g} / \mathrm{m}^{3}$ de Be à moins de $0,001 \mu \mathrm{g} / \mathrm{m}^{3}$ de Be selon la situation.

Dweik propose, pour les sujets sensibilisés, parallèlement à l'arrêt de l'exposition au Be, une radiographie pulmonaire et des EFR mensuels pendant 6 mois puis semestriels (10).

Le diagnostic biologique de sensibilisation est posé en présence d'au moins 2 TPL Be positifs, réalisés de façon optimale dans deux laboratoires différents, sans consensus sur la technique précise de dosage, ni sur le délai ou la fréquence de surveillance des TPL, qui varie de 3 mois à 3 ans, selon la publication (8).

Une sensibilisation a été rapportée dans des intervalles maximaux allant de 2 mois après le début de l'exposition au Be à 40 ans après $(11,12)$. La survenue d'une bérylliose chronique a été décrite dans les 3 mois après le début de l'exposition et jusqu' à 20 ans après la fin de l'exposition $(11,13)$. La concentration urinaire de Be n'est pas utilisée en dépistage.

Le diagnostic de bérylliose chronique est retenu historiquement en présence d’au moins 4 des 6 critères ci-après, dont l'un des deux premiers est obligatoire: 1) exposition documentée au Be, 2) présence de Be dans les prélèvements biologiques, 3) mise en évidence de symptômes cliniques des voies aériennes basses, 4) infiltrat réticulonodulaire visible à la radiographie pulmonaire, 5) altération de la fonction respiratoire 
de type obstructif ou restrictif ou diminution de la DLCO, 6) granulome non caséeux ou infiltrat interstitiel de cellules mononuclées à la biopsie pulmonaire (14). Cependant dans les articles identifiés, le diagnostic de bérylliose chronique est retenu le plus souvent sur l'association de l'exposition au Be, de la positivité du TPL Be sanguin et soit du résultat positif de la biopsie pulmonaire (granulome ou infiltrat lymphocytaire) soit de la positivité du TPL Be effectué sur le lavage broncho-alvéolaire. Dans le bilan effectué à la recherche d'une bérylliose chronique, les EFR incluent généralement une DLCO. Après la radiographie pulmonaire, une tomodensitométrie thoracique n’est pas faite de façon systématique. Les anomalies radiologiques sont plus précocement mises en évidence à l'aide d'une tomodensitométrie thoracique en haute résolution. Cependant il a été rapporté 25\% de patients avec bérylliose chronique avérée et tomodensitométrie thoracique normale (10).

L’algorithme décisionnel suivant (figure 1) a été proposé après discussion. Il débute par une consultation dans l'unité de pathologie professionnelle avec anamnèse professionnelle et médicale, examen clinique, radiographie pulmonaire, EFR et TPL Be sanguin. Selon la présence de symptômes respiratoires, d'anomalie de la radiographie pulmonaire, des EFR ou du TPL Be, la stratégie varie. La tomodensitométrie thoracique est réalisée sans injection de produit de contraste avec coupes millimétriques. Si une fibroscopie bronchique est réalisée, un lavage broncho-alvéolaire est associé avec TPL Be sur le liquide de lavage. Lors du suivi des salariés avec TPL Be positif, après éviction du Be, une surveillance semestrielle puis annuelle du TPL Be est proposée jusqu’à sa négativation. 


\section{Résultats}

Lors de leur premier bilan hospitalier, les 14 hommes âgés de 28 à 56 ans (moyenne : 41 ans) sont exposés au Be depuis 1 à 24 ans (moyenne : 8 ans) ; 8 d'entre eux (57,5\%) présentent un tabagisme chronique persistant, 2 (14\%) ont arrêté de fumer avant le premier TPL Be, et 4 (28,5\%) sont non fumeurs.

Lors de leur prise en charge initiale, aucun des 14 salariés n’a de symptôme respiratoire ni extra-respiratoire évoquant une pathologie débutante liée au Be. Durant ce suivi, aucun ne présente d'anomalie de la capacité de diffusion du monoxyde de carbone, lors des EFR. Onze salariés ont effectué au moins un bilan après la mise en place de l'algorithme soit pour chacun un total de 2, 3 ou 4 bilans, séparés par un intervalle d'1 à 3 ans. Quatre salariés ont présenté 1, 2 ou 3 TPL Be anormaux. Il s’est normalisé chez 3 d'entre eux en 3 à 18 mois, parallèlement à une réduction de l'exposition au risque, liée à une diminution de cette activité dans l'entreprise notamment du fait d'une réduction de nombre de pièces en Be. En outre, il est à noter un renforcement des mesures de décontamination surfacique des pièces et des postes. Le TPL Be des dix autres salariés est resté négatif. Pour huit d'entre eux, on dispose d'un ou de deux bilans réalisés à 1, 2, 4 ou 5 ans d'intervalle.

- Le salarié 1, fraiseur, ex-fumeur de 31 ans, a, 2 ans après le début de son exposition au Be, un TPL Be initial positif, «limite » selon la méthode révisée. Le test est négatif trois mois plus tard, mais le patient signale avoir pris, dans l'intervalle, des corticoïdes oraux en raison d'une infection ORL saisonnière. En dehors de cet épisode infectieux d'évolution favorable, il reste asymptomatique entre les deux TPL Be. En raison de la prévention technique déjà en place dans l'entreprise, des niveaux d'exposition relevés lors des prélèvements « in situ » et de l'exposition intermittente du sujet au Be dans 
l’entreprise, il est proposé de revoir le salarié en consultation et de contrôler son TPL Be un an après le dernier test effectué, ce qui n’a pu être fait en raison du départ du salarié de l'entreprise.

- Le salarié 2, fraiseur de 42 ans, tabagique chronique, présente, 3 ans après le début de son exposition au Be, à quelques mois d'intervalle, deux TPL Be initiaux positifs, « limites» selon la méthode révisée. Une éviction de l’exposition au Be est recommandée. Le bilan est par ailleurs normal à l'exception des EFR en faveur d'une maladie des petites voies aériennes (MPVA). Dix huit mois plus tard, le TPL Be est négatif et le reste un an plus tard, sans modification du bilan clinique et paraclinique. Le salarié signale être resté au même poste avec toutefois une baisse de l'activité d’usinage d'alliage au Be au profit d'autres alliages.

- Le salarié 3, fraiseur de 28 ans, tabagique chronique, a, lors de son deuxième bilan, 7 ans après le début de son exposition au Be, un TPL Be «limite », négatif selon la méthode révisée. Il est contrôlé négatif quelques mois plus tard. Il a été affecté au contrôle des pièces. Deux ans après, le bilan est toujours normal.

- Le salarié 11, 31 ans, effectue les prélèvements atmosphériques et surfaciques en Be de l'entreprise. Il signale ne pas porter de surcombinaison lors de ses déplacements dans l'atelier d’usinage du Be pour effectuer les prélèvements. Il est fumeur et présente, lors du troisième bilan, 6 ans après le début de son exposition au Be, un TPL Be « limite » avec la $1^{\text {ère }}$ méthode, positif selon la méthode révisée. Il a par ailleurs une toux matinale, un examen clinique et une radiographie pulmonaire normaux et des EFR discrètement altérées en faveur d’une MPVA. Le TPL Be est confirmé « limite » quelques mois après et devient positif en 2007, sans modification clinique. L'arrêt de toute exposition au Be est préconisé. Une tomodensitométrie thoracique a été réalisée : elle est normale. 


\section{Discussion}

D’après les données de la littérature la prévalence de sujets sensibilisés au Be varie de 0,8 à 11,8\% selon les études et le secteur industriel (15). Newman rapporte, lors d'un suivi longitudinal réalisé sur une durée moyenne de 4,8 ans (1,7 à 11,6 ans), que 31 \% des 55 salariés de l'industrie de l'armement sensibilisés au Be suivis dans cette étude ont développé une bérylliose chronique (69\% n’ont pas développé de bérylliose chronique) (16). Les postes d'opérateurs (tourneurs, fraiseurs, meuleurs) semblent les plus à risque de sensibilisation (17).

Parmi les 14 salariés exposés au Be suivis dans cette série, on note, chez 4 d'entre eux, un ou plusieurs TPL Be anormaux, normalisés 3 à 18 mois plus tard chez trois sujets. Les 4 hommes chez lesquels des anomalies ont été mises en évidence étaient exposés au Be depuis 2, 3, 6 et 7 ans, alors que la durée d'exposition au Be varie pour les autres de 1 à 24 ans. Il ne semble pas y avoir d'influence de la durée d'exposition au Be sur le résultat du TPL Be.

Pour le salarié 1 , le TPL Be initialement positif est contrôlé négatif, mais 3 mois après corticothérapie : or les corticoïdes seraient susceptibles de négativer le TPL Be (3).

Le TPL Be initialement positif ou «limite» des 2 autres fraiseurs s'est négativé en parallèle à une réduction de l'exposition au Be dans l'entreprise. Une seule étude semble avoir étudié l'effet de la diminution de l'exposition sur l'évolution du TPL Be chez des mineurs ou des salariés employés au traitement du Be (18). Parmi les 82 travailleurs, 13 (16\%) présentent un TPL Be positif alors que la moyenne des 32 mesures de concentrations atmosphériques d'ambiance sur 8 heures est supérieure à 3 fois la VME. Trois ans plus tard, parallèlement à une diminution de l'exposition au Be 
(moyenne des 13 mesures : 0,5 fois la VME), le test est devenu négatif chez 8 des 11 individus retestés et reste positif chez les 3 autres. L'un des trois est resté à un poste associé à une forte exposition au Be (usinage), le deuxième a conservé un poste à faible exposition (encadrement) et le troisième, au poste initial à faible exposition, a quitté l'entreprise, la seule source persistante d'exposition au Be à un niveau dépassant la VME est l'ensachage. Il est à noter que deux salariés dont le TPL Be initial était négatif l’ont positivé trois ans plus tard, donc après la mise en place des mesures de prévention. La méthodologie employée incite à la prudence quant à l’interprétation des résultats de cette étude, notamment parce que le TPL Be initial n’a pas été contrôlé par un deuxième test.

En ce qui concerne le technicien chargé des prélèvements de Be de l'entreprise (sujet 11), son exposition semble avoir diminué depuis son arrivée dans l'entreprise puisque les activités Be ont décru. Des sensibilisations ont pu être rapportées à faible niveau d'exposition (17). Par ailleurs, la façon dont il dit se protéger est peut-être insuffisante, car il semble qu'il ne revête pas systématiquement l'ensemble des EPI spécifiques lorsqu'il se rend dans l'atelier d’usinage du béryllium pour y effectuer les prélèvements atmosphériques et surfaciques. Se pose la question du rôle d'une contamination cutanée. Une étude prospective récente a évoqué cette possibilité. Elle concerne une centaine de céramistes exposés au Be, nouvellement embauchés dans une entreprise dont la prévention technique semble développée. Ces travailleurs ont fait l’objet d’une surveillance régulière à l'inclusion, puis 3, 6, 12, 24 et 48 mois après (19). Les auteurs rapportent que 30\% des sujets testés à l'embauche ont effectué un TPL Be à 24 mois et environ $10 \%$ des sujets à 48 mois et qu'une sensibilisation a été mise en évidence au bout de 24 mois de suivi. Or, dans cette étude, en dépit d’une prévention technique 
développée, une exposition cutanée au Be est rapportée pour le patient sensibilisé. La contribution d’une contamination cutanée a été peu évaluée jusqu’ici. La pénétration cutanée est favorisée en cas de lésion cutanée (4). En outre, il a été montré que des particules de dextran de diamètre inférieur à 1 micron peuvent pénétrer la peau jusqu’au derme (20). Se pose alors la question de la pénétration percutanée de particules de Be de très petite taille. Peu de données humaines sont actuellement disponibles chez des sujets susceptibles d’avoir été sensibilisés au Be par voie cutanée. Une étude pilote récente, axée sur le calcul à partir de modèles mathématiques et la comparaison de l'absorption cutanée et respiratoire du Be a été menée dans l’industrie du recyclage (21). Elle inclut des mesures surfaciques de Be sur la combinaison et la peau de travailleurs exposés au Be. Cette étude montre que lors de l'utilisation de combinaisons en polyester, la concentration en Be retrouvée sur la peau est faible mais quantifiable (9 à 16\% de la quantité de particules retrouvées sur la combinaison), contrairement à ce qui est observé avec les combinaisons en coton, (où la concentration est inférieure au seuil de détection). Enfin, même en présence d'un équipement de protection individuelle, un contact cutané au niveau du visage ne peut être exclu (22).

Par ailleurs, il est suspecté que d'autres caractéristiques conditionnent la biodisponibilité et donc la toxicité du Be (comme le rapport taille/surface, la nature chimique du dérivé incriminé...), de même que des facteurs de risque individuels apparaissent prédisposer au développement d’une sensibilisation et d’une bérylliose chronique tels que certains polymorphismes génétiques au niveau du complexe majeur d’histocompatibilité de classe II ou du gène du TNF $\alpha$ (23, 24). Indépendamment des aspects éthiques que leur réalisation poserait, la valeur prédictive positive de ce dépistage génétique apparaît cependant actuellement insuffisante pour qu'ils soient 
proposés de façon systématique (25).

Parmi les 26 TPL Be réalisés avant septembre 2006 et recalculés pour être aussi présentés selon la méthode révisée, on note 5 différences de résultats entre les deux modes de calcul. Trois résultats positifs selon la méthode initiale sont « limites » avec le deuxième mode de calcul, un résultat « limite » devient négatif et un résultat « limite » devient positif. Cela montre une variabilité des résultats pour un même prélèvement selon la méthode de calcul. Le deuxième mode de calcul semble mathématiquement plus robuste et devrait être utilisé par les biologistes afin que la standardisation des méthodes de dosages permette de comparer les TPL Be entre les patients et au cours du temps. En outre, le contrôle du TPL Be anormal par un deuxième prélèvement distinct, si possible dans un deuxième laboratoire qui utilise des méthodes validées, est très important.

La moitié de nos salariés (7/14) ont été suivis pendant 4 à 6 ans et pendant cette période, 5 d’entre eux ont bénéficié d’au moins 3 bilans cliniques et/ou paracliniques tous les un à trois ans. Les résultats présentés montrent que les modalités de réalisation du suivi annuel seraient à optimiser, la compliance des patients n’étant pas parfaite.

On a vu la rareté des données de suivi de salariés exposés au Be dans la littérature. Dans l'algorithme, nous proposons la réalisation d'une fibroscopie bronchique avec lavage broncho-alvéolaire en fonction du résultat de la tomodensitométrie thoracique, comme c'est le cas dans l'algorithme du Québec. Nous n'avons pas eu, à ce jour, à suivre cet algorithme jusqu'à son terme. Cependant, du fait des résultats observés pour le salarié 11, se pose la question, dans ce type de situation, de la réalisation d'un TPL Be sur le lavage broncho-alvéolaire qui apporterait un argument supplémentaire sur le risque de développer une bérylliose chronique, malgré son caractère plus invasif que celui d’une 
tomodensitométrie thoracique.

\section{Conclusion}

Si le TPL Be a montré son intérêt pour le diagnostic de la bérylliose chronique, en revanche sa place dans le dépistage de la sensibilisation chez les sujets exposés fait encore débat. Les raisons sont les suivantes : sensibilité et spécificité insuffisantes du TPL Be sanguin, grande variabilité inter-et intra-laboratoires qui rend nécessaire la répétition des prélèvements, prévalence de la bérylliose chronique et de la sensibilisation au Be mal connues (26, 27). Le calcul des résultats du TPL Be selon la méthode du département de l'énergie américain semble plus pertinent (8). Ce test devrait idéalement être réalisé par des laboratoires entraînés. Que le but soit le dépistage de la bérylliose chronique ou de sujets sensibilisés, une standardisation de la réalisation du TPL Be et de l'expression de ses résultats semble incontournable. En France, il est à noter qu'il n’y a actuellement, à notre connaissance, qu'un seul laboratoire capable de réaliser ce test biologique en routine. Par ailleurs les conséquences professionnelles et socio-économiques pour les salariés soumis à ce dépistage sont également à considérer. Notre objectif était la mise en place d’une démarche systématisée pour le suivi médical des salariés exposés professionnellement au Be. Quatorze salariés en ont bénéficié avec les difficultés décrites. Un suivi à long terme de populations plus nombreuses pourra apporter des informations supplémentaires et contribuer à préciser le suivi médical le plus approprié. Il est à noter qu'un groupe de travail établi sous l'égide de la Société française de médecine du travail sur la surveillance des sujets exposés au Be est actuellement en cours de réflexion et a pour objectif de déterminer la surveillance à proposer aux personnes exposées. 


\section{Références}

1- Hoover MD. Beryllium properties and uses. IRSST, 2005. http://www.irsst.qc.ca/fr/_publicationirsst_100149.html.

2- Vincent R, Grzebyk M, Savary B. Occupational exposure to beryllium in France. IRSST, 2005. http://www.irsst.qc.ca/fr/_publicationirsst_100149.html.

3- Marchand-Adam S, Valeyre D. Bérylliose pulmonaire chronique : un modèle d'interaction entre environnement et prédisposition génétique (1ère partie). Minéralogie,

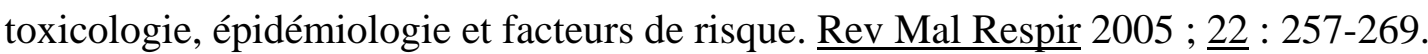

4- Deubner DC, Lowney YW, Paustenbach DJ, Warmerdam J. Contribution of incidental exposure pathways to total beryllium exposures. Appl Occup Environ Hyg $2001 ; \underline{16}: 568-578$

5- Kriebel D, Brain JD, Sprince NL, Kazemi H. The pulmonary toxicity of beryllium.

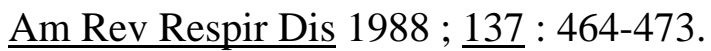

6- IARC. Beryllium, cadmium, mercury and exposures in the glass manufacturing industry. IARC monographs on the evaluation of carcinogenic risks to humans. Volume 58. Lyon, IARC, 1993.

7- Dion C, Perrault G. Summary of good cleanup and decontamination practices for workplaces with beryllium-containing dust. IRSST, 2005, 26p.

8- Frome EL, Newman LS, Cragle DL, Colyer SP, Wambach PF. Identification of an abnormal beryllium lymphocyte proliferation test. Toxicology 2003; 183: 39-56.

9- Institut national de santé publique, Québec. Le test sanguin de prolifération lymphocytaire au béryllium (Be LPT) 2004. http://www.inspq.qc.ca/

10- Dweik R. Diagnosis and management of beryllium sensitization and CBD. IRSST 2005 http://www.irsst.qc.ca/fr/_publicationirsst_100149.html. 
11- Kelleher PC, Martyny JW, Mroz MM, Maier LA, Ruttenber AJ, Young DA, Newman LS. Beryllium particulate exposure and disease relations in a beryllium machining plant. J Occup Environ Med 2001 ; 43 : 238-249.

12- Henneberger PK, Cumro D, Deubner DD, Kent MS, McCawley M, Kreiss K. Beryllium sensitization and disease among long-term and short-term workers in a beryllium ceramics plant. Int Arch Occup Env Health 2001 ; 74: 167-176.

13- Rossman MD. History of beryllium disease. IRSST, 2005. http://www.irsst.qc.ca/fr/_publicationirsst_100149.html.

14- Newman LS, Kreiss K, King TE Jr, Seay S, Campbell PA. Pathologic and immunologic alterations in early stages of beryllium disease. Reexamination of disease definition and natural history. Am Rev Respir Dis 1989 ; 139 : 1479-1486.

15- Marchand-Adam S, Guillon F, Brauner M, Valeyre D. Bérylliose pulmonaire chronique (2ème partie). Pathogénie, expression clinique, prévention et législation. $\underline{\text { Rev }}$

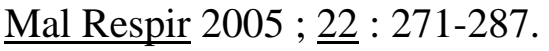

16- Newman LS, Mroz MM, Balkisoon R, Maier LA. Beryllium sensitization progresses to chronic beryllium disease. A longitudinal study of disease risk. Am J

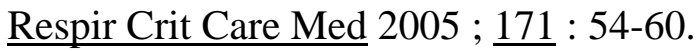

17- Mroz M. Epidemiology of beryllium sensitization and chronic beryllium disease. $\begin{array}{lllll}\text { Overview of recent epidemiologic studies. IRSST, } 2005 & \end{array}$ http://www.irsst.qc.ca/fr/_publicationirsst_100149.html

18- Rom WN, Lockey JE, Bang KM, Dewitt C, Johns RE Jr. Reversible beryllium sensitization in a prospective study of beryllium workers. Arch Environ Health.1983 ; 38: 302-307. 
19- Cummings KJ, Deubner DC, Day GA, Hennenberger PK, Kitt MM, Kent MS, Kreiss K, Schuler C R. Enhanced preventive programme at a beryllium oxide ceramics facility reduces beryllium sensitization among new workers. Occup Environ Med 2007; $\underline{64}: 134-140$.

20- Tinkle SS, Antonini JM, Rich BA, Roberts JR, Salmen R, DePree K, Adkins EJ. Skin as a route of exposure and sensitization in chronic beryllium disease. Environ Health Perspect 2003; 111: 1202-1208.

21- Emond C, Robin JP, Breton R, Philippe S, Zayed J. Dermal exposure to beryllium : a pilot case study. J Toxicol Environ Health 2007; 70:529-533.

22- Day GA, Dufresne A, Stefaniak AB, Schuler CR, Santon ML, Miller WE, Kent MS, Deubner DC, Kreiss K, Hoover MD. Exposure pathway assessment at a copperberyllium alloy facility. Ann Occup Hyg 2007; 511 : 67-80.

23- Richeldi L, Sorentino R, Saltini C. HLA-DPB1 Glutamate 69 : a genetic marker of beryllium disease. Science 1993; 262: 242-244.

24- Saltini C, Richeldi L, Losi M, Amicosante M, Voorter C, van den Berg-Loonen E, Dweik RA, Wiedemann HP, Deubner DC, Tinelli C. Major histocompatibility locus genetic markers of beryllium sensitization and disease. Eur Respir J 2001 ; 18 : 677684.

25- Pillière F, Vincent R. Recherche sur le béryllium : conférence internationale (Montréal, 8-11 mars 2005). Document pour le Médecin du Travail, INRS 2005 ; 103 : 347-356.

26- Borak J, Woolf SH, Fields CA. Use of beryllium lymphocyte proliferation testing for screening of asymptomatic individuals : an evidence-based asssessment. J Occup Environ Med 2006; 48: 937-947. 
27- Pairon JC, Brochard P, Gehanno JF, Letourneux M, Paris C, Ameille J. Pathologies respiratoires professionnelles. In Lemarié E. La pneumologie fondée sur les preuves. Editions Margaux Orange, Paris, 2008 : 511-579.

28- Stange AW, Hilmas DE, Furman FJ, Gatliffe TR. Beryllium sensitization and chronic beryllium disease at a former nuclear weapons facility. Appl Occup Environ Hyg 2001; 16: 405-417.

29- Newman LS, Lloyd J, Daniloff E. The natural history of beryllium sensitization and chronic beryllium disease. Environ Health Perspect 1996 ; 104 (Sup 5): 937-943.

30- Newman LS, Mroz MM, Maier LA, Daniloff EM, Balkisoon R. Efficacy of serial medical surveillance for chronic beryllium disease in a beryllium machining plant. $\underline{\mathrm{J}}$ Occup Environ Med 2001 ; 43: 231-237. 
Tableau 2 : Protocole de surveillance des sujets exposés au Be : informations issues des études de la littérature

\begin{tabular}{|c|c|c|c|}
\hline Références & Fréquence des bilans (période) & Population d'étude & Informations recherchées \\
\hline 28 & $\begin{array}{l}\text { - } 3 \text { ans si TPL }- \text {, } \\
\text { - fréquence non précisée si } 1 \\
\text { seul TPL + ou radio pulmonaire } \\
\text { anormale (maximum } 2 \text { bilans } \\
\text { entre } 1991 \text { et 1997) }\end{array}$ & $\begin{array}{l}\text { sujets exposés } \\
\text { auparavant } \\
\text { ou actuellement au } \\
\text { Be }\end{array}$ & $\begin{array}{l}\text { Questionnaire médico-professionnel, TPL sanguin (si }+: 2^{\text {ème }} \text { prise de } \\
\text { sang ou contrôle du } 1^{\text {er }} \text { TPL sanguin par un autre laboratoire), radio } \\
\text { pulmonaire si anomalie antérieure ou si TPL antérieur limite. } \\
\text { Si sensibilisation au Be ou si } 1 \text { TPL sanguin }+ \text { ou limite ou si suspicion } \\
\text { de BC : fibroscopie bronchique avec LBA et TPL Be dans LBA et } \\
\text { biopsies pulmonaires transbronchiques }\end{array}$ \\
\hline 29 & Annuelle (entre 1986 et 1994) & $\begin{array}{l}\text { Sensibilisation au Be } \\
\text { ou BC }\end{array}$ & $\begin{array}{l}\text { Exposition, clinique, EFR, test d'effort avec GDS, radio pulmonaire, } \\
\text { ECA, fibroscopie bronchique avec LBA et TPL Be dans le LBA et } \\
\text { marqueurs de l'inflammation ; biopsies pulmonaires transbronchiques en } \\
\text { cas de sensibilisation au Be ; TDM avec coupes fines lors du } 1^{\text {er }} \text { bilan }\end{array}$ \\
\hline 30 & $\begin{array}{l}-2 \text { ans ou } \\
\text { l'embauche puis tous les } 2 \text { ans } \\
\text { (maximum } 3 \text { bilans entre } 1995 \text { et } \\
\text { 1999) }\end{array}$ & exposés au Be & $\begin{array}{l}\text { tous les } 2 \text { ans : autoquestionnaire et TPL Be sanguin (répété par même } \\
\text { laboratoire si TPL Be sanguin anormal). } \\
\text { Si plus de } 2 \text { TPL Be sanguins +: évaluation clinique, EFR, test d'effort } \\
\text { avec GDS, fibroscopie bronchique avec LBA et TPL Be dans le LBA et } \\
\text { biopsies pulmonaires transbronchiques }\end{array}$ \\
\hline 16 & $\begin{array}{l}2 \text { ans (maximum } 4 \text { bilans entre } \\
1988 \text { et 1998) }\end{array}$ & Sensibilisation au Be & $\begin{array}{l}\text { EFR, test d'effort, GDS, radio pulmonaire, fibroscopie bronchique avec } \\
\text { LBA et TPL Be dans le LBA et biopsies pulmonaires transbronchiques }\end{array}$ \\
\hline 12 & $\begin{array}{l}6 \text { ans (1992 } \text { et } 1998) \text { ou } \\
\text { ponctuellement si symptômes } \\
\text { pulmonaires ou si radio } \\
\text { pulmonaire annuelle anormale }\end{array}$ & exposés au Be & $\begin{array}{l}\text { questionnaire médico-professionnel et TPL Be sanguin. Si TPL Be }+ \text { : } \\
2^{\text {ème }} \text { TPL Be sanguin ou contrôle du } 1^{\text {er }} \text { TPL Be sanguin par même ou } \\
\text { autre laboratoire. Si } 2 \text { TPL Be sanguins }+: \text { fibroscopie bronchique avec } \\
\text { LBA et TPL Be dans le LBA et biopsies pulmonaires transbronchiques }\end{array}$ \\
\hline 19 & $\begin{array}{l}0,3,6,12,24,48 \text { mois (entre } \\
2000 \text { et 2004) }\end{array}$ & exposés au Be & $\begin{array}{l}\text { TPL Be sanguin. Si + : contrôle par même ou autre laboratoire. Si } 2 \text { TPL } \\
\text { Be sanguins distincts }+ \text { : radio pulmonaire, fibroscopie bronchique avec } \\
\text { LBA et TPL Be dans le LBA et biopsies pulmonaires transbronchiques }\end{array}$ \\
\hline
\end{tabular}

BC : bérylliose chronique, Be : béryllium, ECA : enzyme de conversion de l’angiotensine dans le sang, EFR : épreuves fonctionnelles respiratoires avec spirométrie, boucle débit-volume et capacité de diffusion au monoxyde de carbone, GDS : gazométrie artérielle, TPL Be : test de prolifération lymphocytaire au Be, LBA : lavage broncho-alvéolaire, TDM : tomodensitométrie thoracique, + : positif. 
Figure 1 : algorithme de surveillance des salariés exposés au béryllium

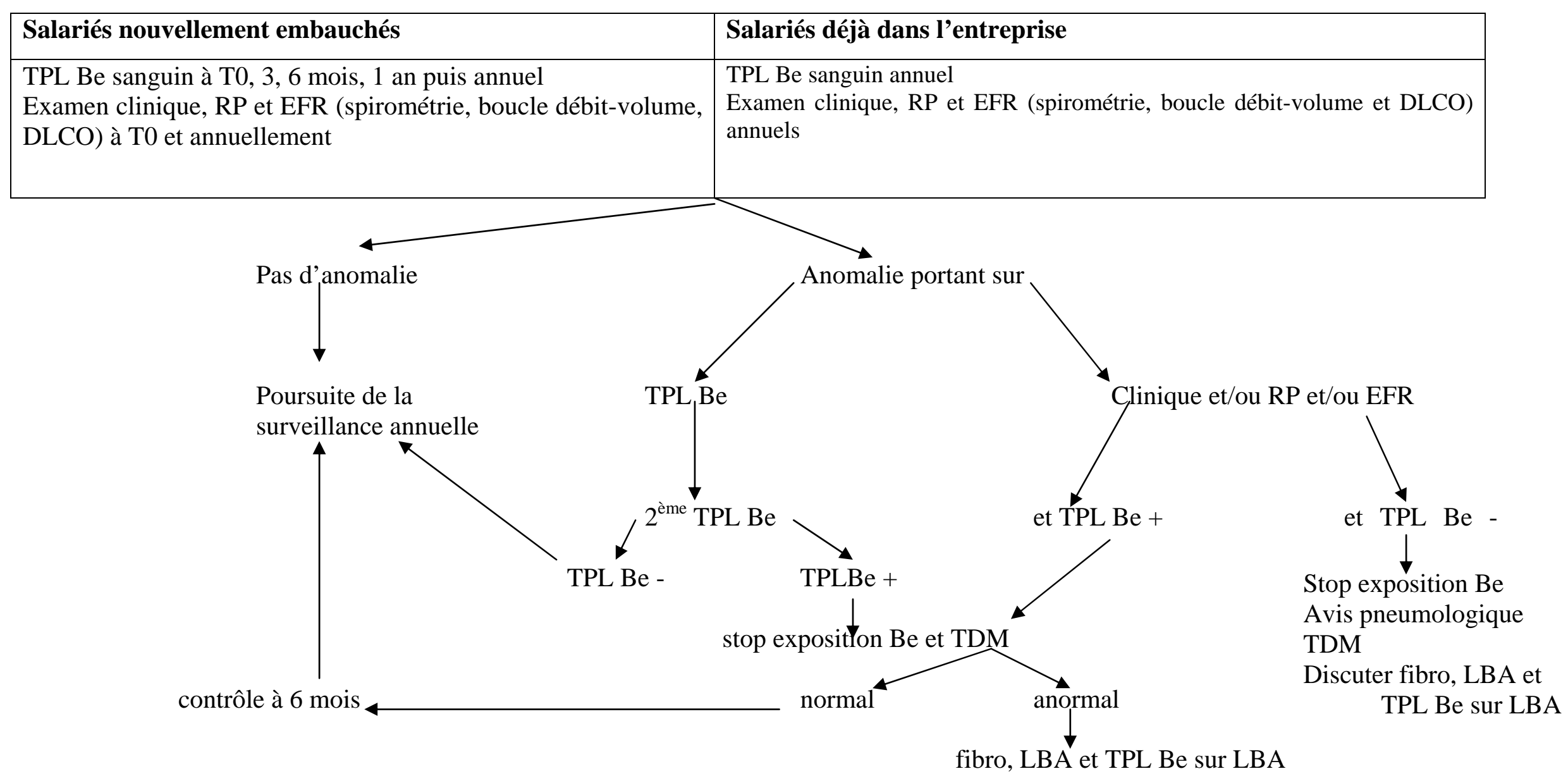

EFR : explorations fonctionnelles respiratoires, DLCO : capacité de diffusion du CO, fibro: fibroscopie bronchique, LBA : lavage bronchoalvéolaire, RP : radiographie pulmonaire, TDM : tomodensitométrie thoracique, TPL Be : test de prolifération lymphocytaire au béryllium ; Be : béryllium, + : positif, - : négatif 
Tableau 1 : Résultats des TPL Be

\begin{tabular}{|c|c|c|c|c|c|c|}
\hline $\begin{array}{l}\text { Sujet } \\
\text { (âge)* }\end{array}$ & année & tabac & $\begin{array}{l}\text { Durée } \\
\text { d'exposition } \\
\text { au Be } \\
\text { (évolution) }\end{array}$ & métier & $\begin{array}{ll}\text { TPL } & \text { Be } \\
\text { sanguin } & \end{array}$ & $\begin{array}{l}\text { TPL Be } \\
\text { méthode révisée }\end{array}$ \\
\hline $1(31)$ & 2004 & $\mathrm{EF}$ & 2 ans & Fraiseur & + puis- & Limite puis - \\
\hline $2(42)$ & $\begin{array}{l}2004 \\
2006 \\
2007\end{array}$ & $\mathrm{~F}$ & $\begin{array}{l}3 \text { ans } \\
\downarrow\end{array}$ & Fraiseur & $\begin{array}{l}+ \text { puis }+ \\
\text { ND } \\
\text { ND }\end{array}$ & $\begin{array}{l}\text { Limite puis Limite } \\
\text { - } \\
\text { - }\end{array}$ \\
\hline $3(28)$ & $\begin{array}{l}2002 \\
2004 \\
2006\end{array}$ & $\mathrm{~F}$ & $\begin{array}{l}5 \text { ans } \\
\downarrow\end{array}$ & $\begin{array}{l}\text { Fraiseur } \\
\text { Fraiseur } \\
\text { Contrôleur }\end{array}$ & $\begin{array}{l}- \\
\text { limite puis - } \\
\text { ND }\end{array}$ & $\begin{array}{l}- \\
\text { - puis - } \\
\text { - }\end{array}$ \\
\hline $4(48)$ & $\begin{array}{l}2005 \\
2006\end{array}$ & $\mathrm{~F}$ & 5 ans & Fraiseur & $\begin{array}{l}- \\
-\end{array}$ & $\begin{array}{l}- \\
-\end{array}$ \\
\hline $5(48)$ & $\begin{array}{l}2002 \\
2006\end{array}$ & EF & $\begin{array}{l}9 \text { ans } \\
\downarrow\end{array}$ & $\begin{array}{l}\text { Ajusteur (atelier Be) } \\
\text { Ajusteur (hors atelier } \\
\text { Be) }\end{array}$ & $-\overline{N D}$ & $\begin{array}{l}- \\
-\end{array}$ \\
\hline $6(31)$ & $\begin{array}{l}2001 \\
2006\end{array}$ & $\mathrm{NF}$ & $\begin{array}{l}4 \text { ans } \\
\downarrow\end{array}$ & Fraiseur & ND & - \\
\hline $7(45)$ & $\begin{array}{l}2004 \\
2006\end{array}$ & NF & $\begin{array}{l}5 \text { ans } \\
\downarrow\end{array}$ & Technicien de contrôle & $-\overline{N D}$ & $\begin{array}{l}- \\
-\end{array}$ \\
\hline $8(52)$ & $\begin{array}{l}2001 \\
2006\end{array}$ & $\mathrm{NF}$ & $\begin{array}{l}24 \text { ans } \\
\downarrow\end{array}$ & Ajusteur & $\begin{array}{l}- \\
\text { ND }\end{array}$ & $\begin{array}{l}- \\
-\end{array}$ \\
\hline $9(40)$ & $\begin{array}{l}2001 \\
2004 \\
2006 \\
\end{array}$ & NF & $\begin{array}{l}20 \text { ans } \\
\downarrow\end{array}$ & $\begin{array}{l}\text { Chef de fabrication / } \\
\text { contrôleur }\end{array}$ & $\begin{array}{l}- \\
- \\
-\end{array}$ & $\begin{array}{l}- \\
- \\
-\end{array}$ \\
\hline $10(48)$ & 2001 & $\mathrm{~F}$ & 15 ans & Tourneur & - & - \\
\hline $11(31)$ & $\begin{array}{l}2001 \\
2004 \\
2006 \\
2007 \\
\end{array}$ & $\mathrm{~F}$ & $\begin{array}{l}1 \text { an } \\
\downarrow\end{array}$ & $\begin{array}{l}\text { Technicien de contrôle: } \\
\text { prélèvements }\end{array}$ & $\begin{array}{l}- \\
- \\
\text { Limite puis ND } \\
\text { ND }\end{array}$ & $\begin{array}{l}- \\
- \\
+ \text { puis Limite } \\
+\end{array}$ \\
\hline $12(30)$ & 2004 & $\mathrm{~F}$ & 6 ans & Technicien & - & - \\
\hline $13(56)$ & $\begin{array}{l}2004 \\
2006\end{array}$ & $\mathrm{~F}$ & $\begin{array}{l}5 \text { ans } \\
\downarrow\end{array}$ & $\begin{array}{l}\text { Fraiseur } \\
\text { Monteur }\end{array}$ & $\begin{array}{l}- \\
-\end{array}$ & $\begin{array}{l}- \\
-\end{array}$ \\
\hline $14(46)$ & $\begin{array}{l}2001 \\
2004 \\
2006\end{array}$ & $\mathrm{~F}$ & $\begin{array}{l}3 \text { ans } \\
\downarrow\end{array}$ & Fraiseur & $\begin{array}{l}- \\
- \\
-\end{array}$ & $\begin{array}{l}- \\
- \\
-\end{array}$ \\
\hline
\end{tabular}

Be : béryllium, $\downarrow$ : réduction de l'exposition au Be, TPL Be : test de prolifération lymphocytaire au béryllium , F : fumeur, NF : non fumeur, EF : ex-fumeur, ND : non disponible, + : test positif, - : test négatif, * : lors du premier TPL Be sanguin 